\title{
A Class of Harmonic Meromorphic Functions of Complex Order
}

\author{
R. Ezhilarasi, K.G. Subramanian and T.V. Sudharsan
}

\begin{abstract}
The seminal work of Clunie and Sheil-Small [3] on harmonic mappings gave rise to studies on subclasses of complex-valued harmonic univalent functions. In this paper a class of harmonic meromorphic functions of the form $\mathrm{f}(\mathrm{z})=\mathrm{h}(\mathrm{z})+\overline{\mathrm{g}(\mathrm{z})},|\mathrm{z}|>1$ of complex order is introduced. It is shown that the functions in this class are sense preserving and univalent outside the unit disk. Sufficient coefficient conditions are obtained for functions in this class which are also shown to be necessary when the co-analytic part $g(\mathrm{z})$ has negative coefficients. We also obtain properties such as distortion bounds, extreme points, convolution and convex combination for this class.
\end{abstract}

Keywords--- Harmonic Functions, Starlike Functions

\section{INTRODUCTION}

$\mathrm{H}$ armonic univalent mappings are known to play an important role in the study of minimal surfaces and have found applications in different fields such as Engineering, Operations research and applied mathematics [2]. Harmonic mappings in a domain $\mathrm{D} \subseteq \mathrm{C}$ are univalent complex valued harmonic functions $\mathrm{f}=\mathrm{u}+$ iv where both $\mathrm{u}$ and $\mathrm{v}$ real harmonic in D. Harmonic univalent mappings have drawn tremendous attention of complex analysts only after the important work of Clunie and Sheil-Small [3] in 1984. Hengartner and Schober [5], [6] in 1986 worked towards finding an appropriate form of the Riemann mapping theorem for harmonic mappings. The works of these function theorists and several other researchers (see for example [7], [11], [12]) gave rise to several problems, conjectures and many intriguing questions. Several classes of complex valued harmonic univalent functions have been introduced and investigated following the basic work of Clunie and Sheil-Small [3]. There are several survey articles and books ([2], [4]) on harmonic mappings and related areas. Hengartner and Schober [7], among other things, investigated the family $\mathrm{M}$ of functions $\mathrm{f}(\mathrm{z})=\mathrm{h}(\mathrm{z})+\overline{\mathrm{g}(\mathrm{z})}$ which are harmonic, meromorphic, orientation preserving and univalent in $\bar{U}=\{z:|z|>1\}$ where

R. Ezhilarasi, Assistant Professor, Department of Mathematics, SIVET College, Chennai - 600 073, India, E-mail : ezhilarasi2008@ymail.com

K.G. Subramanian, Professor, School of Computer Sciences, Universiti Sains Malaysia, 11800 Penang, Malaysia, E-mail : kgsmani1948@yahoo.com

T.V. Sudharsan, Associate Professor, Department of Mathematics, SIVET College, Chennai - 600 073, India, E-mail: tvsudharsan@rediffmail.com $\mathrm{h}(\mathrm{z})=\mathrm{z}+\sum_{\mathrm{n}=1}^{\infty} \mathrm{a}_{\mathrm{n}} \mathrm{z}^{-\mathrm{n}} ; \quad \mathrm{g}(\mathrm{z})=\sum_{\mathrm{n}=1}^{\infty} \mathrm{b}_{\mathrm{n}} \mathrm{z}^{-\mathrm{n}}, \quad \mathrm{z} \in \overline{\mathrm{U}}$

Jahangiri [8] and Jahangiri and Silverman [9] have also investigated harmonic, meromorphic functions which are starlike in $\overline{\mathrm{U}}$.

Here we introduce another class $\mathrm{S}_{\mathrm{H}}(\mathrm{b}, \alpha, \gamma, \mathrm{t})$ of harmonic, meromorphic functions defined as follows:

For $0 \leq \beta<1$, let $\mathrm{S}_{\mathrm{H}}(\mathrm{b}, \alpha, \gamma, \mathrm{t})$ consist of functions $\mathrm{f} \in \mathrm{M}$ so that

$\operatorname{Re}\left\{1+\frac{1}{\mathrm{~b}}\left[\left(\frac{\left(1+\mathrm{e}^{\mathrm{i} \alpha}\right) \mathrm{zf}^{\prime}(\mathrm{z})}{\mathrm{f}_{\mathrm{t}}(\mathrm{z})}-\mathrm{e}^{\mathrm{i} \alpha}\right)-1\right]\right\} \geq \gamma$

where $\mathrm{f}_{\mathrm{t}}(\mathrm{z})=(1-\mathrm{t}) \mathrm{z}+\mathrm{t} \mathrm{f}(\mathrm{z}), 0 \leq \mathrm{t} \leq 1,0 \leq \gamma<1, \alpha$ real and $\mathrm{b}$ a complex number such that $|\mathrm{b}| \leq 1$.

Remark 1.1: The class includes a variety of well-known subclasses for specific values of $\alpha, b$ and $t$.

1. when $b=1, t=1, S_{H}(1, \alpha, \gamma, 1)=M_{\bar{H}}(\gamma)[10]$

2. when $b=1, \mathrm{~S}_{\mathrm{H}}(1, \alpha, \gamma, \mathrm{t})=\mathrm{G}_{\mathrm{H}}(\alpha, \beta, \mathrm{t})[1]$

3. when $\mathrm{b}=1, \alpha=0, \mathrm{t}=1, \mathrm{~S}_{\mathrm{H}}(1,0, \gamma, 1)=\Sigma_{\mathrm{H}}^{*}\left(\frac{1+\beta}{2}\right)[8]$

Also let $\mathrm{S}_{\overline{\mathrm{H}}}(\mathrm{b}, \alpha, \gamma, \mathrm{t})$ be the subclass of $\mathrm{S}_{\mathrm{H}}(\mathrm{b}, \alpha, \gamma, \mathrm{t})$ consisting of functions $\mathrm{f}=\mathrm{h}+\overline{\mathrm{g}}$ in which $\mathrm{h}$ and $\mathrm{g}$ are of the form

$$
\begin{aligned}
& \mathrm{h}(\mathrm{z})=\mathrm{z}+\sum_{\mathrm{n}=1}^{\infty} \mathrm{a}_{\mathrm{n}} \mathrm{z}^{-\mathrm{n}} ; \\
& \mathrm{g}(\mathrm{z})=-\sum_{\mathrm{n}=1}^{\infty} \mathrm{b}_{\mathrm{n}} \mathrm{z}^{-\mathrm{n}}, \quad \mathrm{a}_{\mathrm{n}} \geq 0, \mathrm{~b}_{\mathrm{n}} \geq 0
\end{aligned}
$$

We obtain sufficient coefficient conditions for harmonic meromorphic functions $\mathrm{f}=\mathrm{h}+\overline{\mathrm{g}}$ to be in the class $\mathrm{S}_{\mathrm{H}}(\mathrm{b}, \alpha, \gamma, \mathrm{t})$. We also show that this coefficient condition is also necessary for $\mathrm{f} \in \mathrm{S}_{\overline{\mathrm{H}}}(\mathrm{b}, \alpha, \gamma, \mathrm{t})$. We also obtain distortion bounds, extreme points, convolution condition and convex combination for functions in $\mathrm{S}_{\overline{\mathrm{H}}}(\mathrm{b}, \alpha, \gamma, \mathrm{t})$.

\section{COEFFICIENT CONDITIONS}

First we prove a sufficient condition for harmonic functions in $\mathrm{S}_{\mathrm{H}}(\mathrm{b}, \alpha, \gamma, \mathrm{t})$. 
Theorem 2.1: Let $\mathrm{f}=\mathrm{h}+\overline{\mathrm{g}}$ be so that $\mathrm{h}$ and $\mathrm{g}$ are of the form (1). If

$$
\begin{aligned}
\sum_{n=1}^{\infty}[2 n+t(2-(1-\gamma)|b|)]\left|a_{n}\right| \\
\quad+\sum_{n=1}^{\infty}[2 n-t(2-(1-\gamma)|b|)]\left|b_{n}\right| \leq(1-\gamma)|b|
\end{aligned}
$$

when $0 \leq \gamma<1,0 \leq \mathrm{t} \leq 1, \alpha$ real and $\mathrm{b}$ a non-zero complex number such that $|b| \leq 1$, then $f$ is univalent, sense preserving, harmonic mapping in $\mathrm{U}=\{\mathrm{z}:|\mathrm{z}|<1\}$ and $\mathrm{f} \in \mathrm{S}_{\mathrm{H}}(\mathrm{b}, \alpha, \gamma, \mathrm{t})$.

Proof: Consider the function $\mathrm{f}=\mathrm{h}+\overline{\mathrm{g}}$, where $\mathrm{h}$ and $\mathrm{g}$ are given by (1). In [9] it has been proved that if $\sum_{n=1}^{\infty} n\left|a_{n}\right|+\sum_{n=1}^{\infty} n\left|b_{n}\right| \leq 1,\left|b_{1}\right|<1 \quad$ then $f \quad$ is harmonic, orientation preserving and univalent in $\mathrm{U}$.

For $0 \leq \gamma<1$, we note that

$$
\begin{aligned}
& \mathrm{n} \leq \frac{2 \mathrm{n}+\mathrm{t}(2-(1-\gamma)|\mathrm{b}|)}{(1-\gamma)|\mathrm{b}|} \text { and } \\
& \mathrm{n} \leq \frac{2 \mathrm{n}-\mathrm{t}(2-(1-\gamma)|\mathrm{b}|)}{(1-\gamma)|\mathrm{b}|}
\end{aligned}
$$

Therefore $f$ is harmonic, orientation preserving and univalent in $\overline{\mathrm{U}}$ due to (4). To show that $\mathrm{f} \in \mathrm{S}_{\mathrm{H}}(\mathrm{b}, \alpha, \gamma, \mathrm{t})$ we notice according to (2), we must have $\operatorname{Re}\left[\frac{\mathrm{A}(\mathrm{z})}{\mathrm{B}(\mathrm{z})}\right]>\gamma$ where

$$
\begin{aligned}
A(z)= & b[(1-t) z+t(h(z)+\overline{g(z)})]+\left(1+e^{i \alpha}\right)\left[z h^{\prime}(z)-\overline{z^{\prime}(z)}\right] \\
& -\left(1+e^{i \alpha}\right)[(1-t) z+t(h(z)-\overline{g(z)})] \\
B(z)= & b[(1-t) z+t(h(z)+\overline{g(z)})]
\end{aligned}
$$

Using the fact that $\operatorname{Re}(w) \geq \gamma$ if and only if $|1-\gamma+w| \geq$ $|1+\gamma-w|$ for $0 \leq \gamma<1$ it is enough to show that

$$
|\mathrm{A}(\mathrm{z})+(1-\gamma) \mathrm{B}(\mathrm{z})|-|\mathrm{A}(\mathrm{z})-(1+\gamma) \mathrm{B}(\mathrm{z})| \geq 0
$$

Differentiating $h$ and $g$ and substituting in the above inequality we obtain

$$
\begin{aligned}
&|A(z)+(1-\gamma) B(z)|-|A(z)-(1+\gamma) B(z)| \\
&=\mid\left.\mid(2-\gamma) b-\left(1+e^{i \alpha}\right)\right](1-t) z+b(2-\gamma) \operatorname{th}(z) \\
&+\left(1+e^{i \alpha}\right) z^{\prime}(z)-\left(1+e^{i \alpha}\right) \operatorname{th}(z) \\
&+\overline{b(2-\gamma) \operatorname{tg}(z)-\left(1+e^{i \alpha}\right) z^{\prime}(z)-\left(1+e^{i \alpha}\right) \operatorname{tg}(z)} \mid \\
&-\mid\left[\gamma b+1+e^{i \alpha}\right](1-t) z+\gamma b \operatorname{th}(z) \\
&-\left(1+e^{i \alpha}\right) z^{\prime}(z)+\left(1+e^{i \alpha}\right) \operatorname{th}(z) \\
&+\frac{\operatorname{rbtg}(z)+\left(1+e^{i \alpha}\right) z^{\prime}(z)+\left(1+e^{i \alpha}\right) \operatorname{tg}(z) \mid}{}
\end{aligned}
$$

$$
\begin{gathered}
\geq|b|(2-\gamma)|z|-\sum_{n=1}^{\infty}[2 n+2 t-(2-\gamma)|b| t]\left|a_{n}\right||z|^{-n} \\
\quad-\sum_{n=1}^{\infty}[2 n+(2-\gamma)|b| t-2 t]\left|b_{n}\right||z|^{-n} \\
\quad-\gamma|b||z|-\sum_{n=1}^{\infty}[2 n+\gamma|b| t+2 t]\left|a_{n}\right||z|^{-n} \\
\quad-\sum_{n=1}^{\infty}[2 n-\gamma|b| t-2 t]\left|b_{n}\right||z|^{-n} \\
=2\left\{(1-\gamma)|b||z|-\sum_{n=1}^{\infty}\left[2 n+t(2-(1-\gamma)|b|]\left|a_{n}\right||z|^{-n}\right.\right. \\
-\sum_{n=1}^{\infty}\left[2 n-t(2-(1-\gamma)|b|]\left|b_{n}\right||z|^{-n}\right\} \\
\geq 2\{(1-\gamma)|b| \\
-\sum_{n=1}^{\infty}\left\{\left[2 n+t(2-(1-\gamma)|b|]\left|a_{n}\right|+\left[2 n-t(2-(1-\gamma)|b|]\left|b_{n}\right|\right\}\right\}\right.
\end{gathered}
$$

Now by (4), this last expression is never negative and so $\mathrm{f}$ $\in \mathrm{S}_{\mathrm{H}}(\mathrm{b}, \alpha, \gamma, \mathrm{t})$. We now give an example of a function in the class $\mathrm{S}_{\mathrm{H}}(\mathrm{b}, \alpha, \gamma, \mathrm{t})$.

Example 2.1: The harmonic function $\mathrm{f}=\mathrm{h}+\overline{\mathrm{g}}$ where

$$
\begin{aligned}
& \mathrm{h}(\mathrm{z})=\mathrm{z}+\frac{|\mathrm{b}|(1-\gamma)}{4[2+\mathrm{t}(2-(1-\gamma)|\mathrm{b}|)]} \frac{1}{\mathrm{z}}, \\
& \mathrm{g}(\mathrm{z})=\frac{|\mathrm{b}|(1-\gamma)}{4[2-\mathrm{t}(2-(1-\gamma)|\mathrm{b}|)]} \frac{1}{\bar{z}} .
\end{aligned}
$$

where $0 \leq \gamma<1$ and $0 \leq \mathrm{t} \leq 1$, satisfies the sufficient condition of Theorem 2.1 and hence belongs to the class $\mathrm{S}_{\mathrm{H}}(\mathrm{b}, \alpha, \gamma, \mathrm{t})$.

Next we show that the coefficient condition (4) is also necessary for functions in $\mathrm{S}_{\overline{\mathrm{H}}}(\mathrm{b}, \alpha, \gamma, \mathrm{t})$.

Theorem 2.2: Let $\mathrm{f}=\mathrm{h}+\overline{\mathrm{g}}$ be so that $\mathrm{h}$ and $\mathrm{g}$ are of the form (3). A necessary and sufficient condition for $\mathrm{f}$ to be in $\mathrm{S}_{\overline{\mathrm{H}}}(\mathrm{b}, \alpha, \gamma, \mathrm{t})$ is that

$$
\begin{gathered}
\sum_{n=1}^{\infty}\left\{[2 n+t(2-(1-\gamma)|b|)]\left|a_{n}\right|+[2 n-t(2-(1-\gamma)|b|)]\left|b_{n}\right|\right\} \\
\leq(1-\gamma)|b|
\end{gathered}
$$

Proof: In view of Theorem 2.1, we need only show that $\mathrm{f} \notin \mathrm{S}_{\overline{\mathrm{H}}}(\mathrm{b}, \alpha, \gamma, \mathrm{t})$ if the coefficient inequality (5) does not hold. We note that if $\mathrm{f} \in \mathrm{S}_{\overline{\mathrm{H}}}(\mathrm{b}, \alpha, \gamma, \mathrm{t})$ we must have 


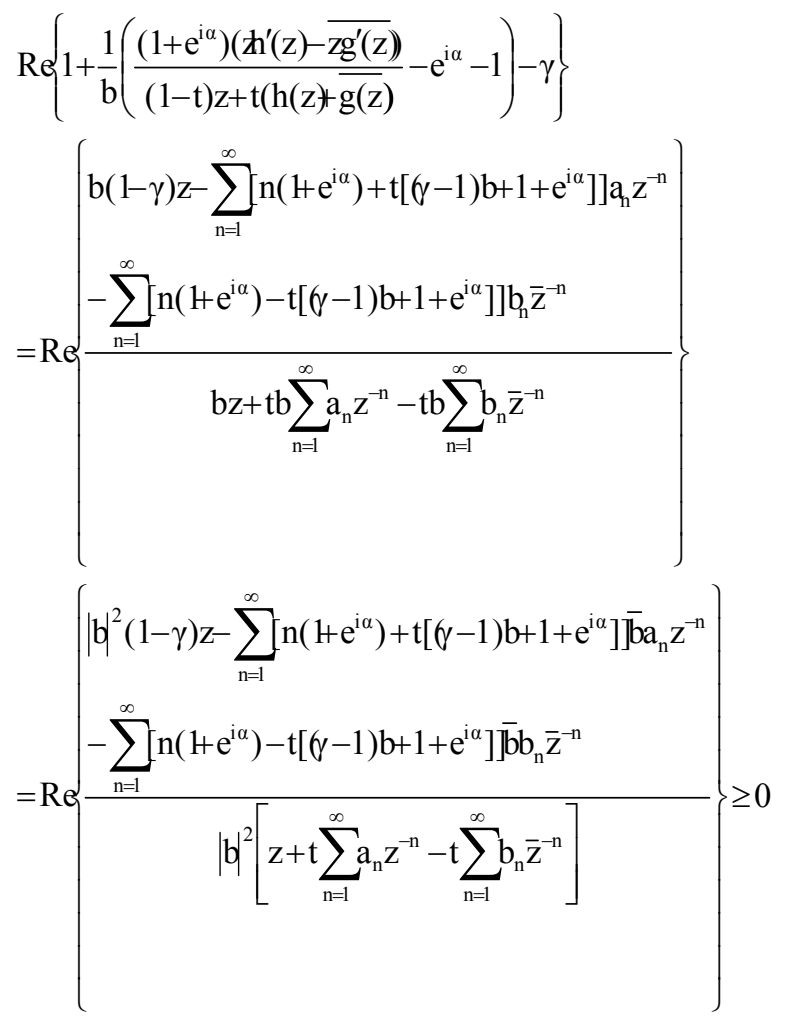

This inequality must hold for all $\mathrm{z} \in \overline{\mathrm{U}}$ and for all real $\alpha$ and any $b$ such that $0<|b|<1$. Letting $z=r>1, \alpha=0$ and $b$ real and positive so that $|\mathrm{b}|=\mathrm{b}$, we have

$$
\operatorname{Re}\left\{\begin{array}{l}
|b|^{2}(1-\gamma)-\sum_{n=1}^{\infty}\left[2 n+t[2-(1-\gamma) b] \bar{b} a_{n} r^{-(n+1)}\right. \\
|b|^{2}\left(1+t \sum_{n=1}^{\infty} a_{n} r^{-(n+1)}-t \sum_{n=1}^{\infty} b_{n} r^{-(n+1)}\right)
\end{array}=\frac{A(r)}{B(r)} \geq 0\right.
$$

If the condition (5) does not hold, then $\mathrm{A}(\mathrm{r})$ is negative for $r$ sufficiently close to 1 . Thus there exists a $z_{0}=r>1$ for which the quotient $\frac{\mathrm{A}(\mathrm{r})}{\mathrm{B}(\mathrm{r})}$ is negative. This contradicts that $\frac{\mathrm{A}(\mathrm{r})}{\mathrm{B}(\mathrm{r})} \geq 0$ and so the proof is complete.

The distortion bounds for functions in $\mathrm{S}_{\overline{\mathrm{H}}}(\mathrm{b}, \alpha, \gamma, \mathrm{t})$ are given by Theorem 2.3.

Theorem 2.3: If $\mathrm{f} \in \mathrm{S}_{\overline{\mathrm{H}}}(\mathrm{b}, \alpha, \gamma, \mathrm{t})$ then

$$
\mathrm{r}-|\mathrm{b}|(1-\gamma) \mathrm{r}^{-1} \leq|\mathrm{f}(\mathrm{z})| \leq \mathrm{r}+|\mathrm{b}|(1-\gamma) \mathrm{r}^{-1}, \quad|\mathrm{z}|=\mathrm{r}>1 .
$$

Proof: We prove the right hand inequality. The argument for the left hand inequality is similar and hence is omitted. Let $\mathrm{f} \in \mathrm{S}_{\overline{\mathrm{H}}}(\mathrm{b}, \alpha, \gamma, \mathrm{t})$. Taking the absolute value of $\mathrm{f}$ we obtain

$$
\begin{aligned}
& \mid f(z)=\left|z+\sum_{n=1}^{\infty} a_{n} z^{-n}-\sum_{n=1}^{\infty} b_{n} z^{-n}\right| \\
& \leq r+\sum_{n=1}^{\infty}\left[\left|a_{n}\right|+\left|b_{n}\right|\right) r^{-n} \\
&\left.\leq r+\sum_{n=1}^{\infty}[2 n+t(2-(1-\gamma) \mid b)]\left|a_{n}\right|+[2 n+t(2-(1-\gamma) \mid b)]\left|b_{n}\right|\right\} r^{-1} \\
& \leq r+\mid b(1-\gamma) r^{-1} . \\
& \text { III. } \quad \text { EXTREME POINTS }
\end{aligned}
$$

\section{EXTREME POINTS}

We use the coefficient bounds obtained in section 2 to determine the extreme points for functions in $\mathrm{S}_{\overline{\mathrm{H}}}(\mathrm{b}, \alpha, \gamma, \mathrm{t})$.

Theorem 3.1: if $\in \mathrm{S}_{\overline{\mathrm{H}}}(\mathrm{b}, \alpha, \gamma, \mathrm{t})$ if and only if $\mathrm{f}$ can be expressed as $\mathrm{f}=\sum_{\mathrm{n}=0}^{\infty}\left(\mathrm{x}_{\mathrm{n}} \mathrm{h}_{\mathrm{n}}+\mathrm{y}_{\mathrm{n}} \mathrm{g}_{\mathrm{n}}\right)$ where $\mathrm{z} \in \overline{\mathrm{U}}$,

$$
\begin{aligned}
& \mathrm{h}_{0}(\mathrm{z})=\mathrm{z}, \quad \mathrm{h}_{\mathrm{n}}(\mathrm{z})=\mathrm{z}+\frac{|\mathrm{b}|(1-\gamma)}{2 \mathrm{n}+\mathrm{t}(2-(1-\gamma)|\mathrm{b}|} \mathrm{z}^{-\mathrm{n}} \quad(\mathrm{n}=1,2, \ldots) \\
& \mathrm{g}_{0}(\mathrm{z})=\mathrm{z}, \quad \mathrm{g}_{\mathrm{n}}(\mathrm{z})=\mathrm{z}-\frac{|\mathrm{b}|(1-\gamma)}{2 \mathrm{n}-\mathrm{t}(2-(1-\gamma)|\mathrm{b}|)}(\overline{\mathrm{z}})^{-\mathrm{n}} \quad(\mathrm{n}=1,2, \ldots)
\end{aligned}
$$

and

$$
\sum_{n=0}^{\infty}\left(x_{n}+y_{n}\right)=1, \quad x_{n} \geq 0, y_{n} \geq 0
$$

Proof: Note that for f we may write

$$
\begin{aligned}
f(z)= & \sum_{n=0}^{\infty}\left(x_{n} h_{n}+y_{n} g_{n}\right) \\
= & x_{0} h_{0}+y_{0} g_{0}+\sum_{n=1}^{\infty}\left(x_{n}\left[z+\frac{\mid b(1-\gamma)}{2 n+t(2-(1-\gamma / b)} z^{-n}\right]\right. \\
& \left.+y_{n}\left[z-\frac{\mid b(1-\gamma)}{2 n-t(2-(1-\gamma|b|)}(z)^{-n}\right]\right) \\
= & \sum_{n=0}^{\infty}\left(x_{n}+y_{n}\right) z+\sum_{n=1}^{\infty} \frac{\mid b(1-\gamma)}{2 n+t(2-(1-\gamma) b)} x_{n} z^{-n} \\
& -\sum_{n=1}^{\infty} \frac{\mid b(1-\gamma)}{2 n-t(2-(1-\gamma) b \mid b)} y_{n}(\bar{z})^{-n}
\end{aligned}
$$

Now by Theorem 2.2

$$
\begin{aligned}
& \sum_{n=1}^{\infty}\left\{[2 n+t(2-(1-\gamma)|b|)]\left[\frac{|b|(1-\gamma) x_{n}}{2 n+t(2-(1-\gamma)|b|)}\right]\right. \\
& \left.\quad+[2 n-t(2-(1-\gamma)|b|)]\left[\frac{|b|(1-\gamma) y_{n}}{2 n-t(2-(1-\gamma)|b|)}\right]\right\} \\
& =|b|(1-\gamma) \sum_{n=1}^{\infty}\left(x_{n}+y_{n}\right) \leq|b|(1-\gamma) .
\end{aligned}
$$

Conversely, suppose that $\mathrm{f} \in \mathrm{S}_{\overline{\mathrm{H}}}(\mathrm{b}, \alpha, \gamma, \mathrm{t})$ then 


$$
\sum_{n=1}^{\infty}\left[\frac{2 n+t(2-(1-\gamma)|b|)}{|b|(1-\gamma)} a_{n}+\frac{2 n-t(2-(1-\gamma)|b|)}{|b|(1-\gamma)} b_{n}\right] \leq 1 .
$$

Setting

$$
\begin{gathered}
x_{n}=\frac{2 n+t(2-(1-\gamma)|b|)}{|b|(1-\gamma)} a_{n}, \\
y_{n}=\frac{2 n-t(2-(1-\gamma)|b|)}{|b|(1-\gamma)} b_{n}, \quad 0 \leq x_{0} \leq 1 . \\
y_{0}=1-x_{0}-\sum_{n=1}^{\infty}\left(x_{n}+y_{n}\right),
\end{gathered}
$$

we obtain $f(z)=\sum_{n=0}^{\infty}\left(x_{n} h_{n}+y_{n} g_{n}\right)$ as required.

\section{Convolution AND CONVEX Combination}

In this section we show that the class $\mathrm{S}_{\overline{\mathrm{H}}}(\mathrm{b}, \alpha, \gamma, \mathrm{t})$ is invariant under convolution and convex combinations of its members.

For harmonic functions

$$
\begin{aligned}
& f(z)=z+\sum_{n=1}^{\infty} a_{n} z^{-n}-\sum_{n=1}^{\infty} b_{n}(\bar{z})^{-n}, \\
& F(z)=z+\sum_{n=1}^{\infty} A_{n} z^{-n}-\sum_{n=1}^{\infty} B_{n}(\bar{z})^{-n} .
\end{aligned}
$$

We define the convolution of $\mathrm{f}$ and $\mathrm{F}$ as

$$
\begin{aligned}
(f * F)(z) & =f(z) * F(z) \\
& =z+\sum_{n=1}^{\infty} a_{n} A_{n} z^{-n}-\sum_{n=1}^{\infty} b_{n} B_{n}(\bar{z})^{-n}
\end{aligned}
$$

Theorem 4.1: For $0 \leq \beta \leq \gamma \leq 1$. Let $\mathrm{f} \in \mathrm{S}_{\overline{\mathrm{H}}}(\mathrm{b}, \alpha, \gamma, \mathrm{t})$ and $\mathrm{F} \in \mathrm{S}_{\overline{\mathrm{H}}}(\mathrm{b}, \alpha, \beta, \mathrm{t})$.

Then $\mathrm{f}^{*} \mathrm{~F} \in \mathrm{S}_{\overline{\mathrm{H}}}(\mathrm{b}, \alpha, \gamma, \mathrm{t}) \subset \mathrm{S}_{\overline{\mathrm{H}}}(\mathrm{b}, \alpha, \beta, \mathrm{t})$.

Proof: Suppose $\mathrm{f}$ and $\mathrm{F}$ are so that $\mathrm{f} * \mathrm{~F}$ is given by the above convolution.

Since $f \in S_{\bar{H}}(b, \alpha, \gamma, t)$ and $F \in S_{\bar{H}}(b, \alpha, \beta, t)$, the coefficients of $f$ and $F$ must satisfy conditions given by Theorem 2.2. So far the coefficients of $\mathrm{f}^{*} \mathrm{~F}$ we can write

$$
\begin{aligned}
& \sum_{n=1}^{\infty}\left\{[2 n+t(2-(1-\gamma)|b|)] a_{n} A_{n}+[2 n-t(2-(1-\gamma)|b|)] b_{n} B_{n}\right\} \\
& \leq \sum_{n=1}^{\infty}\left\{[2 n+t(2-(1-\gamma)|b|)] a_{n}+[2 n-t(2-(1-\gamma) \mid b)] b_{n}\right\}
\end{aligned}
$$

The right hand side of the above inequality is bounded by $\mathrm{b}(1-\gamma)$ because $\mathrm{f} \in \mathrm{S}_{\overline{\mathrm{H}}}(\mathrm{b}, \alpha, \gamma, \mathrm{t})$.

Thus f* $\mathrm{F} \in \mathrm{S}_{\overline{\mathrm{H}}}(\mathrm{b}, \alpha, \gamma, \mathrm{t}) \subset \mathrm{S}_{\overline{\mathrm{H}}}(\mathrm{b}, \alpha, \beta, \mathrm{t})$.

Finally we examine the convex combinations of $\mathrm{S}_{\overline{\mathrm{H}}}(\mathrm{b}, \alpha, \gamma, \mathrm{t})$.
Theorem 4.2: The family $\mathrm{S}_{\overline{\mathrm{H}}}(\mathrm{b}, \alpha, \gamma, \mathrm{t})$ is closed under convex combination.

\section{Proof: Suppose}

$$
\begin{gathered}
f_{i}(z)=z+\sum_{n=1}^{\infty} a_{i_{n}} z^{-n}-\sum_{n=1}^{\infty} b_{i_{n}}(\bar{z})^{-n} \in S_{\bar{H}}(b, \alpha, \gamma, t) \text { where } \\
a_{i_{n}} \geq 0, \quad b_{i_{n}} \geq 0 \text { and } i=1,2,3, \ldots \text { Then by Theorem 2.2, } \\
\sum_{n=1}^{\infty}\left[[2 n+t(2-(1-\gamma)|b|)] a_{i_{n}}+[2 n-t(2-(1-\gamma)|b|)] b_{i_{n}}\right] \leq|b|(1-\gamma) \\
\text { For } \sum_{i=1}^{\infty} t_{i}=1 \text { and } 0 \leq t_{i} \leq 1, \text { the convex combinations of } f_{i},
\end{gathered}
$$
may be written as

$$
\sum_{i=1}^{\infty} \mathrm{t}_{\mathrm{i}} \mathrm{f}_{\mathrm{i}}(\mathrm{z})=\mathrm{z}+\sum_{\mathrm{n}=1}^{\infty}\left(\sum_{\mathrm{i}=1}^{\infty} \mathrm{t}_{\mathrm{i}} \mathrm{a}_{\mathrm{i}_{\mathrm{n}}}\right) \mathrm{z}^{-\mathrm{n}}-\sum_{\mathrm{n}=1}^{\infty}\left(\sum_{\mathrm{i}=1}^{\infty} \mathrm{t}_{\mathrm{i}} \mathrm{b}_{\mathrm{i}_{\mathrm{n}}}\right)(\overline{\mathrm{z}})^{-\mathrm{n}}
$$

Thus, $\sum_{\mathrm{i}=1}^{\infty} \mathrm{t}_{\mathrm{i}} \mathrm{f}_{\mathrm{i}}(\mathrm{z}) \in \mathrm{S}_{\overline{\mathrm{H}}}(\mathrm{b}, \alpha, \gamma, \mathrm{t})$, since

$$
\begin{aligned}
& \sum_{\mathrm{n}=1}^{\infty}\left[\left[2 \mathrm{n}+\mathrm{t}(2-(1-\gamma) \mid \mathrm{b}) \cdot\left(\sum_{\mathrm{i}=1}^{\infty} \mathrm{t}_{\mathrm{i}} \mathrm{a}_{\mathrm{i}_{\mathrm{n}}}\right)\right.\right. \\
& \left.\quad+[2 \mathrm{n}-\mathrm{t}(2-(1-\gamma) \mathrm{b})\}\left(\sum_{\mathrm{i}=1}^{\infty} \mathrm{t}_{\mathrm{i}} \mathrm{b}_{\mathrm{i}_{\mathrm{n}}}\right)\right] \\
& =\sum_{\mathrm{i}=1}^{\infty} \mathrm{t}_{\mathrm{i}}\left\{\sum_{\mathrm{n}=1}^{\infty}[2 \mathrm{n}+\mathrm{t}(2-(1-\gamma)|\mathrm{b}|)] \mathrm{a}_{\mathrm{i}_{\mathrm{n}}}+[2 \mathrm{n}+\mathrm{t}(2-(1-\gamma) \mid \mathrm{b})] \mathrm{b}_{\mathrm{i}_{\mathrm{n}}}\right\} \\
& \leq \sum_{\mathrm{i}=1}^{\infty} \mathrm{t}_{\mathrm{i}}|\mathrm{b}|(1-\gamma)=\mid \mathrm{b}(1-\gamma) .
\end{aligned}
$$

\section{CONCLUSION}

In this paper an attempt has been made to introduce and investigate some properties for a new subclass of harmonic meromorphic functions of complex order. Based on this work, further useful study on different subclasses of harmonic univalent functions can be established.

\section{REFERENCES}

[1] B. Adolf Stephen, P. Nirmaladevi, T.V. Sudharsan and K.G. Subramanian, A class of meromorphic functions with negative coefficients, Chamchuri J. Maths., Vol. 1, No. 1, Pp.83-90, 2009.

[2] O.P. Ahuja, Planar harmonic univalent and related mappings, J. Inequal. Pure Appl. Math., Vol. 6, No. 4, Art-122, 2005.

[3] J. Clunie and T. Sheil-Small, Harmonic univalent functions, Ann. Acad. Sci. Fenn. Ser. A, I Math., Vol. 9, Pp. 3-25, 1984.

[4] P.L. Duren, Harmonic mappings in the plane, Cambridge University Press, 2004.

[5] W. Hengartner and G. Schober, Harmonic mappings with given dilations, J. London Math. Soc., Vol. 33, No. 3, Pp. 473-483, 1986.

[6] W. Hengartner and G. Schober, On the boundary behavior of orientation-preserving harmonic mappings, Complex Variables Theory Appl., Vol. 5, No. 2-4, Pp. 197-208, 1986.

[7] W. Hengartner and G. Schober, Univalent harmonic functions, Trans. Amer. Math. Soc., Vol. 299, Pp. 1-31, 1987.

[8] J.M. Jahangiri, Harmonic meromorphic starlike functions, Bull. Korean Math. Soc., Vol. 37, Pp. 291-301, 2000. 
[9] J.M. Jahangiri and H. Silverman, Meromorphic univalent harmonic functions with negative coefficients, Bull. Korean Math. Soc., Vol. 36, Pp. 763-770, 1999.

[10] T. Rosy, B. Adolf Stephen, K.G. Subramanian and J.M. Jahangiri, A class of harmonic meromorphic functions, Tamkang J. Math., Vol. 33, Pp. 5-9, 2002.

[11] T. Sheil-Small, Constants for planar harmonic mappings, J. London Math. Soc., Vol. 42, No. 2, Pp. 237-248, 1990.

[12] T.J. Suffridge, Harmonic univalent polynomials, Complex Variables Theory Appl., Vol. 35, No. 2, Pp. 93-107, 1998. 